TRANSACTIONS OF THE

AMERICAN MATHEMATICAL SOCIETY

Volume 359, Number 11, November 2007, Pages 5345-5363

S 0002-9947(07)04192-X

Article electronically published on May 11, 2007

\title{
ASYMPTOTIC DISTRIBUTION OF THE LARGEST OFF-DIAGONAL ENTRY OF CORRELATION MATRICES
}

\author{
WANG ZHOU
}

\begin{abstract}
Suppose that we have $n$ observations from a $p$-dimensional population. We are interested in testing that the $p$ variates of the population are independent under the situation where $p$ goes to infinity as $n \rightarrow \infty$. A test statistic is chosen to be $L_{n}=\max _{1 \leq i<j \leq p}\left|\rho_{i j}\right|$, where $\rho_{i j}$ is the sample correlation coefficient between the $i$-th coordinate and the $j$-th coordinate of the population. Under an independent hypothesis, we prove that the asymptotic distribution of $L_{n}$ is an extreme distribution of type $G_{1}$, by using the Chen-Stein Poisson approximation method and the moderate deviations for sample correlation coefficients. As a statistically more relevant result, a limit distribution for $l_{n}=\max _{1 \leq i<j \leq p}\left|r_{i j}\right|$, where $r_{i j}$ is Spearman's rank correlation coefficient between the $i$-th coordinate and the $j$-th coordinate of the population, is derived.
\end{abstract}

\section{INTRODUCTION}

Suppose $(X, Y),\left(X_{i}, Y_{i}\right), i=1, \ldots, n$ are independent and identically distributed (i.i.d.) random vectors from a bivariate distribution $F(x, y)$. For measuring the closeness of two random variables $X$ and $Y$, we use Pearson's sample correlation coefficient, which is defined by

$$
\rho_{X Y}=\frac{\sum_{i=1}^{n}\left(X_{i}-\bar{X}\right)\left(Y_{i}-\bar{Y}\right)}{\sqrt{\sum_{i=1}^{n}\left(X_{i}-\bar{X}\right)^{2} \sum_{i=1}^{n}\left(Y_{i}-\bar{Y}\right)^{2}}},
$$

where $\bar{X}=\sum_{i=1}^{n} X_{i} / n$ and $\bar{Y}=\sum_{i=1}^{n} Y_{i} / n$. Obviously, $\rho_{X Y}$ is a natural estimator of the population correlation coefficient,

$$
\rho=\mathbf{E}(X-\mathbf{E} X)(Y-\mathbf{E} Y) / \sqrt{\mathbf{E}(X-\mathbf{E} X)^{2}(Y-\mathbf{E} Y)^{2}} .
$$

In the case of a bivariate normal distribution, $\rho_{X Y}$ is the maximum likelihood estimator of $\rho$. In addition, it is a commonly used statistic to test the null hypothesis that the two variables $X, Y$ are independent, i.e.,

$$
\begin{array}{ll}
H_{0}: & \rho=0, \\
H_{1}: & \rho \neq 0 .
\end{array}
$$

Received by the editors April 25, 2005 and, in revised form, September 5, 2005.

2000 Mathematics Subject Classification. Primary 60F05, 62G20,62H10.

Key words and phrases. Sample correlation matrices, Spearman's rank correlation matrices, Chen-Stein method, moderate deviations.

The author was supported in part by grants R-155-000-035-112 and R-155-050-055-133/101 at the National University of Singapore.

(C)2007 American Mathematical Society Reverts to public domain 28 years from publication 
Although many authors have studied the distribution of $\rho_{X Y}$, see Hotelling [ [6] and the references therein, the best present-day usage in dealing with sample correlation coefficients is based on R. A. Fisher's work published in 1915; see $[3]$.

Now suppose we have a $p$-dimensional distribution with mean $\mu$ and covariance matrix $\Sigma$. In the last three or four decades, due to the fast development of modern technology, in many research areas, including signal processing, network security, image processing, genetics, stock marketing and other economic problems, we are faced with the task of analyzing data with ever increasing dimension $p$. Naturally, one may ask how to test the independence among the $p$ components of the population. For example, air pollutant levels are influenced by several meteorological conditions, such as wind speed, temperature, humidity, insolation, etc. The relationship of air pollutant levels to meteorological conditions can be described by regression models. But an essential part of constructing regression models is to check that all the interested meteorological conditions are independent or uncorrelated.

Under the additional normality assumption, Johnstone 9] derived the asymptotic distribution of the largest eigenvalue of the sample covariance matrices to study the test hypothesis $H_{0}: \Sigma=I$ assuming $\mu=0$. On the other hand, Jiang 7 proposed to use a more intuitive independence test statistic, the largest off-diagonal entry of the sample correlation matrices, to test $H_{0}$ assuming at least a 30th moment of the population.

Before proceeding further, let us introduce some notation. Let $\left(X_{k 1}, \ldots, X_{k p}\right)$, $k=1, \ldots, n$, be a sample of $n$ independent observations taken from a nondegenerate $p$-variate population. Let $\mathbf{X}_{i}=\left(X_{1 i}, \ldots, X_{n i}\right)^{T}, i=1, \ldots, p$, be columns of the $n \times p$ matrix $\mathcal{X}_{n}=\left(X_{k i}\right), k=1, \ldots, n, i=1, \ldots, p$. Hence $\mathcal{X}_{n}=\left(\mathbf{X}_{1}, \mathbf{X}_{2}, \ldots, \mathbf{X}_{p}\right)$. Introduce the average $\bar{X}_{i}=\sum_{k=1}^{n} X_{k i} / n$. We write $\mathbf{X}_{i}-\bar{X}_{i}$ for $\mathbf{X}_{i}-\bar{X}_{i} e$, where $e=(1,1, \ldots, 1)^{T} \in \mathbf{R}^{n}$. Then, $\rho_{i j}$, the sample correlation coefficient between $\mathbf{X}_{i}$ and $\mathbf{X}_{j}$ is defined by

$$
\rho_{i j}=\frac{\left(\mathbf{X}_{i}-\bar{X}_{i}\right)^{T}\left(\mathbf{X}_{j}-\bar{X}_{j}\right)}{\left\|\mathbf{X}_{i}-\bar{X}_{i}\right\|\left\|\mathbf{X}_{j}-\bar{X}_{j}\right\|}, \quad 1 \leq i<j \leq p
$$

where $\|\cdot\|$ is the Euclidean norm. If we assume that the columns of $\mathcal{X}_{n}$ are independent, all the $\rho_{i j}$ should be close to 0 . In other words, the biggest offdiagonal entry of the sample correlation matrix $\Gamma_{n}=\left(\rho_{i j}\right)$,

$$
L_{n}=\max _{1 \leq i<j \leq p}\left|\rho_{i j}\right|
$$

has to be small. So we can use $L_{n}$ as an independence test statistic. Under the i.i.d. assumption, Jiang [7] found an asymptotic distribution of $L_{n}$.

Theorem A (Jiang, [7). Suppose that $\mathbf{E}|X|^{30+\epsilon}<\infty$ for some $\epsilon>0$. If $n / p \rightarrow \gamma$, then as $n \rightarrow \infty$ for any $y \in \mathbf{R}$,

$$
\mathbf{P}\left(n L_{n}^{2}-a_{n} \leq y\right) \rightarrow \exp \left(-\left(\gamma^{2} \sqrt{8 \pi}\right)^{-1} e^{-y / 2}\right),
$$

where $a_{n}=4 \log n-\log \log n$.

Jiang's result makes it possible to test independence of components of vectors in large dimensional data problems when strong structural assumptions on the population, such as normality, are not present. But the conditions of his theorem are too restrictive. For example, a $(30+\epsilon)$ th moment is needed to get the asymptotic 
distribution of $L_{n}$. So is it possible to reduce the high order moment requirements? On the other hand, we have no guarantee that the ratio of the sample size to the dimension will converge in practice. Sometimes it may happen that the dimension increases much slower than the sample size. Clearly, one may ask what we can do in those situations. In this paper, we try to answer the above mentioned questions through the following theorem.

Theorem 1.1. Assume that the entries of the matrix $\mathcal{X}_{n}=\left(X_{k i}\right)$ are i.i.d. Let $p=p_{n}$ satisfy

$$
\lim _{n \rightarrow \infty} p=\infty, \limsup _{n \rightarrow \infty} p / n<\infty .
$$

Write $b_{n}=4 \log p-\log \log p$. Then

$$
\lim _{n \rightarrow \infty} \mathbf{P}\left(n L_{n}^{2}-b_{n} \leq y\right)=\exp \left(-K e^{-y / 2}\right), \quad K=(8 \pi)^{-1 / 2},
$$

provided that

$$
\lim _{x \rightarrow \infty} x^{6} \mathbf{P}\left(\left|X X^{\prime}\right|>x\right)=0,
$$

where $X$ and $X^{\prime}$ are independent copies of $X_{11}$. In particular, (1.6) holds if

$$
\mathbf{E} X^{6}<\infty .
$$

We shall make the following remarks:

(1). Using the Chebyshev inequality, we can see that the condition $\mathbf{E} X^{6}<\infty$ is stronger than the weak moment assumption (1.6) for the product $X X^{\prime}$. Through Example 1 in Section 4, we explain that the weak moment condition (1.6) seems to be optimal.

(2). The limit distributions in (1.5) and (1.3) are different. This is due to the different choice of centering constants. Our choice of centering constants has an advantage that the limit distribution is independent of the unknown parameters, which is really useful in statistical applications. Of course, under Jiang's conditions, the relations (1.5) and (1.3) are mathematically equivalent, since in this case, $\lim _{n \rightarrow \infty}\left(a_{n}-b_{n}\right)=4 \log \gamma$.

(3). To prove Theorem 1.1 we use the Poisson approximation technique, which was suggested by Barbour and Eagleson [2, and self-normalized moderate deviations studied by Shao [10, Wang and Jing [14, Shao [11, Jing, Shao and Wang 8.

Now we can see that the sample correlation coefficient is still useful in large dimensional statistical inference if high order moment assumptions are violated. But if strong structural assumptions on the population, such as normality, are not present, the sample correlation coefficient does not retain much interest because $\rho$ is not invariant under rearrangement of the values of the independent random variables. More precisely, if $Z=\psi(X)$, where $\psi$ is one-to-one and measurable, the correlation coefficient between $X$ and $Y$ may be very different from that between $Z$ and $Y$. Another disadvantage of the sample correlation coefficient is that $\rho=0$ does not necessarily imply the independence. Therefore other measures of independence can be of interest. For the independence test problems, it is Spearman [13] who introduced Spearman's rank correlation coefficient into statistics.

For a column $\mathbf{X}_{i}=\left(X_{1 i}, \ldots, X_{n i}\right)^{T}$, we order the elements $X_{1 i}, \ldots, X_{n i}$ of $\mathbf{X}_{i}$ from least to greatest, and let $Q_{k i}$ denote the rank of $X_{k i}$. Replacing the matrix 
$\mathcal{X}_{n}=\left(X_{k i}\right)$ by the matrix $\left(Q_{k i}\right)$, similar to (1.2), we can define the so-called Spearman rank correlation coefficient between $\mathbf{X}_{i}$ and $\mathbf{X}_{j}$ as

$$
\begin{aligned}
r_{i j} & =\frac{\sum_{k=1}^{n} Q_{k i} Q_{k j}-\frac{1}{n}\left(\sum_{k=1}^{n} Q_{k i}\right)\left(\sum_{k=1}^{n} Q_{k j}\right)}{\sqrt{\sum_{k=1}^{n}\left(Q_{k i}-\bar{Q}_{i}\right)^{2} \sum_{k=1}^{n}\left(Q_{k j}-\bar{Q}_{j}\right)^{2}}} \\
& =\frac{12}{n\left(n^{2}-1\right)} \sum_{k=1}^{n} Q_{k i} Q_{k j}-\frac{3(n+1)}{n-1}
\end{aligned}
$$

where $\bar{Q}_{i}=\bar{Q}_{j}=(n+1) / 2$. All the $r_{i j}$ constitute Spearman's rank correlation matrix

$$
\mathcal{R}_{n}=\left(r_{i j}\right)_{1 \leq i, j \leq p}
$$

The most important point is that the distribution of $r_{i j}$ does not depend on nuisance parameters. Much information on $r_{i j}$ can be found in Hájek, Šidák and Sen [4].

As a statistically more relevant result than Theorem 1.1, we give the following Theorem 1.2 for the largest off-diagonal entry of $\mathcal{R}_{n}$,

$$
l_{n}=\max _{1 \leq i<j \leq p}\left|r_{i j}\right| .
$$

Although $l_{n}$ is distribution-free, it is hard to get the exact distribution of $l_{n}$ when $n$ is large. In order to make statistical inference based on $l_{n}$, it is essential to obtain the asymptotic distribution of $l_{n}$.

Theorem 1.2. Assume that $\mathbf{X}_{1}, \ldots, \mathbf{X}_{p}$ are independent, and all the entries of $\mathcal{X}_{n}$ are continuous random variables. If $p=p_{n}$ satisfy

$$
\lim _{n \rightarrow \infty} p=\infty, \quad \limsup _{n \rightarrow \infty} p / n<\infty,
$$

then

$$
\lim _{n \rightarrow \infty} \mathbf{P}\left(n l_{n}^{2}-b_{n} \leq y\right)=\exp \left(-K e^{-y / 2}\right), \quad K=(8 \pi)^{-1 / 2},
$$

with $b_{n}=4 \log p-\log \log p$.

In Theorem 1.2, we assume that all the entries of $\mathcal{X}_{n}$ are continuous random variables in order to avoid ties among the observations. Otherwise, the number of ties must be taken into account. As a consequence, the expectation and the variance under the null (independence) hypothesis need adjustment. We refer to Hollander and Wolfe [5] for more details. For the adjusted $r_{i j}$, Theorem 1.2 still holds.

The paper is organized as follows. We will prove the main theorems in Section 2. Some technical lemmas are deferred to Section 3. A counterexample is presented in Section 4 to explain that the weak moment condition (1.6) seems to be optimal.

\section{Proofs of MAIN THEOREMS}

We first present a lemma about Poisson approximation, which is a special case of Theorem 1 in Arratia, Goldstein and Gordon [1. In the proofs of the main theorems, we rely heavily on it. 
Lemma 2.1. Let $\mathbf{B}$ be an index set and $\left\{B_{\alpha}, \alpha \in \mathbf{B}\right\}$ be a set of subsets of $\mathbf{B}$, i.e., $B_{\alpha} \subset \mathbf{B}$ for each $\alpha \in \mathbf{B}$. Also let $\left\{\eta_{\alpha}, \alpha \in \mathbf{B}\right\}$ be random variables. For a given $t \in \mathbf{R}$, set $\lambda=\sum_{\alpha \in \mathbf{B}} \mathbf{P}\left(\eta_{\alpha}>t\right)$. Then

$$
\left|\mathbf{P}\left(\max _{\alpha \in \mathbf{B}} \eta_{\alpha} \leq t\right)-e^{-\lambda}\right| \leq\left(1 \wedge \lambda^{-1}\right)\left(b_{1}+b_{2}+b_{3}\right)
$$

where

$$
\begin{aligned}
b_{1} & =\sum_{\alpha \in \mathbf{B}} \sum_{\beta \in B_{\alpha}} \mathbf{P}\left(\eta_{\alpha}>t\right) \mathbf{P}\left(\eta_{\beta}>t\right), \\
b_{2} & =\sum_{\alpha \in \mathbf{B}} \sum_{\alpha \neq \beta \in B_{\alpha}} \mathbf{P}\left(\eta_{\alpha}>t, \eta_{\beta}>t\right), \\
b_{3} & =\sum_{\alpha \in \mathbf{B}} \mathbf{E}\left|\mathbf{P}\left(\eta_{\alpha}>t \mid \sigma\left(\eta_{\beta}, \beta \notin B_{\alpha}\right)\right)-\mathbf{P}\left(\eta_{\alpha}>t\right)\right|,
\end{aligned}
$$

where $\sigma\left(\eta_{\beta}, \beta \notin B_{\alpha}\right)$ is the $\sigma$-algebra generated by $\left\{\eta_{\beta}, \beta \notin B_{\alpha}\right\}$. In particular, if $\eta_{\alpha}$ is independent of $\left\{\eta_{\beta}, \beta \notin B_{\alpha}\right\}$ for each $\alpha$, then $b_{3}=0$.

Before we prove Theorem 1.1, let us illustrate the main steps of the proof. We first show that it suffices to consider $L_{n}$ based on the truncated random variables. See (2.1). Then we replace sample variances, the denominator of each correlation coefficient, by population variances. See (2.4). The next step is to truncate the products $\hat{X}_{k i} \hat{X}_{k j}$. See (2.5). Finally, we apply Lemma 2.1 to get the asymptotic distribution.

For notational convenience, we write

$$
t_{p}=\sqrt{4 \log p-\log \log p+y}
$$

and

$$
t_{p n}=\sqrt{4 \log p-\log \log p+y+\epsilon_{n}}
$$

where $\epsilon_{n} \rightarrow 0$ as $n \rightarrow \infty$. Throughout the paper, for an arbitrary set $\Omega, \Omega^{c}$ denotes the complementary set of $\Omega$.

Proof of Theorem 1.1. Since the sample correlation coefficient is scale and shift invariant, we can assume that

$$
\mathbf{E} X=0, \quad \mathbf{E} X^{2}=1 .
$$

Define

$$
\hat{X}_{k i}=X_{k i} \mathbf{I}\left(\left|X_{k i}\right| \leq \sqrt{n} / \log ^{2} n\right), \hat{\mathbf{X}}_{i}=\left(\hat{X}_{1 i}, \ldots, \hat{X}_{n i}\right)^{T}, \overline{\hat{X}_{i}}=\sum_{k=1}^{n} \hat{X}_{k i} / n,
$$

and

$$
\hat{\rho}_{i j}=\frac{\left(\hat{\mathbf{X}}_{i}-\overline{\hat{X}_{i}}\right)^{T}\left(\hat{\mathbf{X}}_{j}-\overline{\hat{X}_{j}}\right)}{\left\|\hat{\mathbf{X}}_{i}-\overline{\hat{X}_{i}}\right\|\left\|\hat{\mathbf{X}}_{j}-\overline{\hat{X}_{j}}\right\|}, \hat{L}_{n}=\max _{1 \leq i<j \leq p}\left|\hat{\rho}_{i j}\right|,
$$

where $1 \leq i<j \leq p, 1 \leq k \leq n$. Then, for $\kappa<1$,

$$
\begin{aligned}
\left|\mathbf{P}\left(n L_{n}^{2} \leq t_{p}^{2}\right)-\mathbf{P}\left(n \hat{L}_{n}^{2} \leq t_{p}^{2}\right)\right| & \leq \mathbf{P}\left(\max _{1 \leq k \leq n, 1 \leq i \leq p}\left|X_{k i}\right|>\sqrt{n} / \log ^{2} n\right) \\
& \leq n p \mathbf{P}\left(\left|X_{12}\right|>\sqrt{n} / \log ^{2} n\right) \\
& \leq n p(\log n)^{12-2 \kappa} \mathbf{E}|X|^{6-\kappa} / n^{3-\kappa / 2} \rightarrow 0
\end{aligned}
$$


as $p \rightarrow \infty$, where in the last step we used Lemma 3.1 Let us deal with $\hat{L}_{n}$.

Write

$$
\hat{L}_{1 n}=\max _{1 \leq i<j \leq p}\left|\frac{\left(\hat{\mathbf{X}}_{i}-\overline{\hat{X}_{i}}\right)^{T}\left(\hat{\mathbf{X}}_{j}-\overline{\hat{X}_{j}}\right)}{n\left(1-1 /(\log n)^{2}\right)}\right|
$$

and

$$
\hat{L}_{2 n}=\max _{1 \leq i<j \leq p}\left|\frac{\left(\hat{\mathbf{X}}_{i}-\overline{\hat{X}_{i}}\right)^{T}\left(\hat{\mathbf{X}}_{j}-\overline{\hat{X}_{j}}\right)}{n\left(1+1 /(\log n)^{2}\right)}\right| .
$$

Using the basic inequality

$$
\mathbf{P}\left(\Theta_{1}\right) \leq \mathbf{P}\left(\Theta_{1} \cap \Theta_{2}\right)+\mathbf{P}\left(\Theta_{1} \cap \Theta_{2}^{c}\right),
$$

with

$$
\Theta_{1}=\left(n \hat{L}_{n}^{2} \leq t_{p}^{2}\right) \text { and } \Theta_{2}=\left(\max _{1 \leq i \leq p}\left|\left\|\hat{\mathbf{X}}_{i}-\overline{\hat{X}}_{i}\right\|^{2}-n\right| \leq n /(\log n)^{2}\right),
$$

and noting that $\hat{L}_{2 n}^{2} \leq \hat{L}_{n}^{2}$ if $\Theta_{2}$ is true, we have

$$
\mathbf{P}\left(n \hat{L}_{n}^{2} \leq t_{p}^{2}\right) \leq \mathbf{P}\left(n \hat{L}_{2 n}^{2} \leq t_{p}^{2}\right)+\mathbf{P}\left(\max _{1 \leq i \leq p}\left|\left\|\hat{\mathbf{X}}_{i}-\overline{\hat{X}}_{i}\right\|^{2}-n\right|>n /(\log n)^{2}\right) .
$$

On the other hand, since $\hat{L}_{1 n}^{2} \geq \hat{L}_{n}^{2}$ if $\Theta_{2}$ is true, we have

$$
\mathbf{P}\left(\Theta_{1}\right) \geq \mathbf{P}\left(\Theta_{1} \cap \Theta_{2}\right) \geq \mathbf{P}\left(\Theta_{3} \cap \Theta_{2}\right) \geq \mathbf{P}\left(\Theta_{3}\right)-\mathbf{P}\left(\Theta_{2}^{c}\right),
$$

where $\Theta_{3}=\left(n \hat{L}_{1 n}^{2} \leq t_{p}^{2}\right)$.

Combining the above inequalities gives

$$
\begin{aligned}
& \mathbf{P}\left(n \hat{L}_{1 n}^{2} \leq t_{p}^{2}\right)-\mathbf{P}\left(\max _{1 \leq i \leq p}\left|\left\|\hat{\mathbf{X}}_{i}-\overline{\hat{X}_{i}}\right\|^{2}-n\right|>n /(\log n)^{2}\right) \\
\leq & \mathbf{P}\left(n \hat{L}_{n}^{2} \leq t_{p}^{2}\right) \\
\leq & \mathbf{P}\left(n \hat{L}_{2 n}^{2} \leq t_{p}^{2}\right)+\mathbf{P}\left(\max _{1 \leq i \leq p}\left|\left\|\hat{\mathbf{X}}_{i}-\overline{\hat{X}_{i}}\right\|^{2}-n\right|>n /(\log n)^{2}\right) .
\end{aligned}
$$

By (3.20) of Lemma 3.5.

$$
\begin{aligned}
& \mathbf{P}\left(\max _{1 \leq i \leq p}\left|\left\|\hat{\mathbf{X}}_{i}-\overline{\hat{X}_{i}}\right\|^{2}-n\right|>n /(\log n)^{2}\right) \\
& \leq p \mathbf{P}\left(\left|\left\|\hat{\mathbf{X}}_{1}-\overline{\hat{X}_{1}}\right\|^{2}-n\right|>n /(\log n)^{2}\right) \\
& \leq p n^{-M} \rightarrow 0
\end{aligned}
$$

as $n \rightarrow \infty$. Also note that

$$
t_{p}^{2}\left(1 \pm 1 /(\log n)^{2}\right)=t_{p}^{2}+o(1)
$$

as $n \rightarrow \infty$. It follows from (2.2)-(2.3), in order to prove the theorem, that it suffices to prove the following:

$$
\mathbf{P}\left(n \bar{L}_{n}^{2} \leq t_{p}^{2}\right) \rightarrow \exp \left(-K e^{-y / 2}\right)
$$

as $p \rightarrow \infty$, where

$$
\bar{L}_{n}=\max _{1 \leq i<j \leq p}\left|\left(\hat{\mathbf{X}}_{i}-\overline{\hat{X}_{i}}\right)^{T}\left(\hat{\mathbf{X}}_{j}-\overline{\hat{X}_{j}}\right) / n\right| .
$$


Now for $\epsilon$ satisfying (3.33), define

$$
\begin{gathered}
\xi_{k i j}=\hat{X}_{k i} \hat{X}_{k j} \mathbf{I}\left(\left|\hat{X}_{k i} \hat{X}_{k j}\right| \leq \epsilon \sqrt{n}\right), 1 \leq k \leq n, \\
y_{i j}=\frac{\sum_{k=1}^{n} \xi_{k i j}-n \overline{\hat{X}}_{i} \overline{\hat{X}_{j}}}{\sqrt{n}}, 1 \leq i<j \leq p,
\end{gathered}
$$

and

$$
W_{n}=\max _{1 \leq i<j \leq p}\left|y_{i j}\right|
$$

For $W_{n}$, we have

$$
\begin{aligned}
\left|\mathbf{P}\left(n \bar{L}_{n}^{2} \leq t_{p}^{2}\right)-\mathbf{P}\left(W_{n}^{2} \leq t_{p}^{2}\right)\right| & \leq \mathbf{P}\left(\max _{1 \leq k \leq n, 1 \leq i<j \leq p}\left|X_{k i} X_{k j}\right|>\epsilon \sqrt{n}\right) \\
& \leq n p^{2} \mathbf{P}\left(\left|X_{12} X_{13}\right|>\epsilon \sqrt{n}\right) \\
& \rightarrow 0
\end{aligned}
$$

as $p \rightarrow \infty$, where in the last inequality, we used the conditions (1.6) and (1.4). So we may turn to consider $W_{n}$.

We will apply Lemma 2.1 to prove

$$
\mathbf{P}\left(\max _{1 \leq i<j \leq p}\left|y_{i j}\right| \leq t_{p}\right) \rightarrow \exp \left(-K e^{-y / 2}\right)
$$

as $p \rightarrow \infty$.

Take $\mathbf{B}=\{(i, j): 1 \leq i<j \leq p\}$. For $\alpha=(i, j) \in \mathbf{B}$, set $\eta_{\alpha}=\left|y_{i j}\right|$ and $B_{\alpha}=\{(k, l)$ : one of $k$ and $l=i$ or $j$, but $(k, l) \neq \alpha\}$. We first calculate $\lambda=\lambda_{n}$ in Lemma 2.1. Since $\left\{y_{i j}:(i, j) \in \mathbf{B}\right\}$ are identically distributed,

$$
\lambda_{n}=\frac{p^{2}-p}{2} \mathbf{P}\left(\left|y_{12}\right|>t_{p}\right)=\frac{\exp (-y / 2)}{\sqrt{8 \pi}}(1+o(1))
$$

as $p \rightarrow \infty$, where the last equality is from Lemma 3.2 ,

Notice that $y_{\alpha}$ is independent of $\left\{y_{\beta}: \beta \in \mathbf{B} \backslash B_{\alpha}\right\}$ for any $\alpha \in \mathbf{B}$. To complete the proof of (2.8), by Lemma 2.1, we need to show that $b_{1} \rightarrow 0$ and $b_{2} \rightarrow 0$. But

$$
b_{1} \leq \frac{1}{2}\left(p^{2}-p\right) 2 p \mathbf{P}\left(\left|y_{12}\right|>t_{p}\right)^{2}=O(1 / n)
$$

by Lemma 3.2, Also Lemma 3.4 gives

$$
\begin{aligned}
b_{2} & \leq p\left(p^{2}-p\right) \mathbf{P}\left(\left|y_{12}\right|>t_{p},\left|y_{13}\right|>t_{p}\right) \\
& \leq p\left(p^{2}-p\right)\left(\mathbf{P}\left(\left|y_{12}+y_{13}\right|>2 t_{p}\right)+\mathbf{P}\left(\left|y_{12}-y_{13}\right|>2 t_{p}\right)\right) \\
& \rightarrow 0 .
\end{aligned}
$$

Hence (2.8) is verified. So we have completed the proof.

Proof of Theorem 1.2. We apply Lemma 2.1 directly to prove Theorem 1.2

Take $\mathbf{B}=\{(i, j): 1 \leq i<j \leq p\}$. For $\alpha=(i, j) \in \mathbf{B}$, set $\eta_{\alpha}=\sqrt{n}\left|r_{i j}\right|$ and $B_{\alpha}=\{(k, l)$ : one of $k$ and $l=i$ or $j$, but $(k, l) \neq \alpha\}$. We first calculate $\lambda=\lambda_{n}$ in Lemma 2.1. Since $\left\{r_{i j}:(i, j) \in \mathbf{B}\right\}$ are identically distributed,

$$
\lambda_{n}=\sum_{1 \leq i<j \leq p} \mathbf{P}\left(\sqrt{n}\left|r_{i j}\right|>t_{p}\right)=\frac{p^{2}-p}{2} \mathbf{P}\left(\sqrt{n}\left|r_{12}\right|>t_{p}\right) .
$$


By (1.8),

$$
r_{12}=\frac{12}{n\left(n^{2}-1\right)} \sum_{k=1}^{n} Q_{k 1} Q_{k 2}-\frac{3(n+1)}{n-1},
$$

which can be reexpressed as

$$
r_{12}=\frac{12}{n\left(n^{2}-1\right)} \sum_{i=1}^{n} i R_{i}-\frac{3(n+1)}{n-1},
$$

where $R_{i}$ is some $Q_{k 2}$ such that $Q_{k 1}=i$. Now applying Lemma 3.6 to (2.9), we have

$$
\lambda_{n}=\frac{\exp (-y / 2)}{\sqrt{8 \pi}}(1+o(1))
$$

as $n \rightarrow \infty$.

Notice that $r_{\alpha}$ is independent of $\left\{r_{\beta}: \beta \in \mathbf{B} \backslash B_{\alpha}\right\}$ for any $\alpha \in \mathbf{B}$. To complete the proof, by Lemma 2.1, we need to show that $b_{1} \rightarrow 0$ and $b_{2} \rightarrow 0$. But

$$
b_{1} \leq \frac{1}{2}\left(p^{2}-p\right) 2 p \mathbf{P}^{2}\left(\sqrt{n}\left|r_{12}\right|>t_{p}\right)=O(1 / n)
$$

by Lemma 3.6, and

$$
\begin{aligned}
b_{2} & \leq p\left(p^{2}-p\right) \mathbf{P}\left(\sqrt{n}\left|r_{12}\right|>t_{p}, \sqrt{n}\left|r_{13}\right|>t_{p}\right) \\
& =p\left(p^{2}-p\right) \mathbf{E P}_{1}^{2}\left(\sqrt{n}\left|r_{12}\right|>t_{p}\right),
\end{aligned}
$$

where $\mathbf{P}_{1}$ means the conditional probability given $\mathbf{X}_{1}$. For Spearman's correlation coefficient, $\mathbf{P}_{1}\left(\sqrt{n}\left|r_{12}\right|>t_{p}\right)=\mathbf{P}\left(\sqrt{n}\left|r_{12}\right|>t_{p}\right)$ a.s. Applying Lemma 3.6 again gives $b_{2} \rightarrow 0$. So we have completed the proof.

\section{Technical Lemmas}

Let $\left\{\xi, \eta, \xi_{k}, \eta_{k}, \eta_{k}^{\prime}, k=1,2, \ldots, n\right\}$ be i.i.d. random variables with mean 0 and variance 1 . Also suppose

$$
\sup _{x>0} x^{6} \mathbf{P}(|\xi \eta|>x)<\infty .
$$

Lemma 3.1. Under conditions (3.1), we have

$$
\mathbf{E}|\xi|^{6-\kappa}<\infty, \mathbf{E}|\xi \eta|^{6-\kappa}<\infty, \text { for all } 0<\kappa<1 .
$$

The above Lemma 3.1 is well known for one random variable. For the product, just note that $\mathbf{E}|\xi \eta|^{6-\kappa}=\mathbf{E}|\xi|^{6-\kappa} \mathbf{E}|\eta|^{6-\kappa}$, due to the i.i.d. assumption. So we omit its proof.

Now we introduce the following truncated random variables:

$$
\hat{\xi}=\xi \mathbf{I}\left(|\xi| \leq d_{n}\right), \hat{\eta}=\eta \mathbf{I}\left(|\eta| \leq d_{n}\right), \hat{\eta}^{\prime}=\eta^{\prime} \mathbf{I}\left(\left|\eta^{\prime}\right| \leq d_{n}\right)
$$

with

$$
d_{n}=\sqrt{n} /(\log n)^{2} \text { or } \quad d_{n}=n^{1 / 3}
$$

and

$$
\hat{\xi}_{i}=\xi_{i} \mathbf{I}\left(\left|\xi_{i}\right| \leq d_{n}\right), \hat{\eta}_{i}=\eta_{i} \mathbf{I}\left(\left|\eta_{i}\right| \leq d_{n}\right), \hat{\eta}_{i}^{\prime}=\eta_{i}^{\prime} \mathbf{I}\left(\left|\eta_{i}^{\prime}\right| \leq d_{n}\right) \text {, for } i=1,2, \ldots, n \text {. }
$$


So we truncate all the variables at two levels. One is $\sqrt{n} /(\log n)^{2}$, which is used in the proof of Theorem 1.1. The other one is $n^{1 / 3}$, which we use in Example 1 of Section 4. The means of $\hat{\xi}_{i}, \hat{\eta}_{i}$ and $\hat{\eta}_{i}^{\prime}$ are denoted by

$$
\overline{\hat{\xi}}=\sum_{i=1}^{n} \hat{\xi}_{i} / n, \overline{\hat{\eta}}=\sum_{i=1}^{n} \hat{\eta}_{i} / n, \overline{\hat{\eta}}^{\prime}=\sum_{i=1}^{n} \hat{\eta}_{i}^{\prime} / n \text {. }
$$

Define, for $\epsilon$ satisfying (3.33),

$$
\begin{gathered}
z=\hat{\xi} \hat{\eta} \mathbf{I}(|\hat{\xi} \hat{\eta}| \leq \epsilon \sqrt{n}), z^{\prime}=\hat{\xi} \hat{\eta}^{\prime} \mathbf{I}\left(\left|\hat{\xi} \hat{\eta}^{\prime}\right| \leq \epsilon \sqrt{n}\right), \\
z_{i}=\hat{\xi}_{i} \hat{\eta}_{i} \mathbf{I}\left(\left|\hat{\xi}_{i} \hat{\eta}_{i}\right| \leq \epsilon \sqrt{n}\right), z_{i}^{\prime}=\hat{\xi}_{i} \hat{\eta}_{i}^{\prime} \mathbf{I}\left(\left|\hat{\xi}_{i} \hat{\eta}_{i}^{\prime}\right| \leq \epsilon \sqrt{n}\right), \text { for } i=1,2, \ldots, n .
\end{gathered}
$$

Truncating $z$ and $z_{i}$ again, we have

$$
\hat{z}=z \mathbf{I}\left(|z| \leq \sqrt{n} /(\log n)^{2}\right), \hat{z}_{i}=z_{i} \mathbf{I}\left(\left|z_{i}\right| \leq \sqrt{n} /(\log n)^{2}\right), \text { for } i=1,2, \ldots, n .
$$

The sums of $z_{i}, z_{i}^{\prime}, \hat{z}_{i}$ are denoted by

$$
S=\sum_{i=1}^{n} z_{i}, \quad S^{\prime}=\sum_{i=1}^{n} z_{i}^{\prime}, \quad \hat{S}=\sum_{i=1}^{n} \hat{z}_{i}
$$

The following Lemma 3.2 is used to calculate $\lambda$ and $b_{1}$ of Lemma 2.1. The main idea of proving Lemma 3.2 is to replace the population variance by the sample variance so that we can use the self-normalized moderate deviations.

Lemma 3.2. For the above truncated random variables,

$$
\mathbf{P}\left(|(S-n \overline{\hat{\xi}} \overline{\hat{\eta}}) / \sqrt{n}|>t_{p}\right) /\left(\frac{e^{-y / 2}}{\sqrt{2 \pi}} p^{-2}\right) \rightarrow 1,
$$

and for any $A>1$,

$$
\mathbf{P}(|S / \sqrt{n}|>A \sqrt{\log p})=o\left(p^{-A^{2} / 4}\right)
$$

as $n \rightarrow \infty$.

Proof. The Bernstein inequality implies, for any $0<\delta_{0}<1 / 2$, that

$$
\mathbf{P}\left(|\overline{\hat{\xi}}-\mathbf{E} \hat{\xi}|>n^{\delta_{0}-1 / 2}\right) \leq 2 \exp \left(-\frac{n^{2 \delta_{0}}}{2 \operatorname{Var} \hat{\xi}+\frac{2}{3} \epsilon \sqrt{n} n^{\delta_{0}-1 / 2}}\right) \leq n^{-c}
$$

with $c>2$ for $n$ sufficiently large, which combined with $|\mathbf{E} \hat{\xi}| \leq n^{-(5-\kappa) / 3} \mathbf{E}|\xi|^{6-\kappa}$ implies that

$$
\begin{aligned}
\mathbf{P}\left(|S / \sqrt{n}|-4 n^{2 \delta_{0}-1 / 2}>t_{p}\right)-n^{-c} & \leq \mathbf{P}\left(|(S-n \overline{\hat{\xi}} \overline{\hat{\eta}}) / \sqrt{n}|>t_{p}\right) \\
& \leq \mathbf{P}\left(|S / \sqrt{n}|+4 n^{2 \delta_{0}-1 / 2}>t_{p}\right)+n^{-c}
\end{aligned}
$$

for $n$ sufficiently large and $\kappa<1$. Let $\delta_{0} \in(0,1 / 4)$. Then $2 \delta_{0}-1 / 2<0$, which implies that

$$
\left(t_{p} \pm 4 n^{2 \delta-1 / 2}\right)^{2}-t_{p}^{2} \rightarrow 0
$$

as $n \rightarrow \infty$. Hence, in order to prove (3.2), by (3.4) and (3.5) it suffices to prove

$$
\mathbf{P}\left(|S / \sqrt{n}|>t_{p n}\right) /\left(\frac{e^{-y / 2}}{\sqrt{2 \pi}} p^{-2}\right) \rightarrow 1
$$


as $n \rightarrow \infty$. Since

$$
\begin{aligned}
& \mathbf{P}\left(|S / \sqrt{n}|>t_{p n}\right)-\mathbf{P}\left(|\hat{S} / \sqrt{n}|>t_{p n}\right) \\
& =\mathbf{P}\left(|S / \sqrt{n}|>t_{p n}, \max _{1 \leq i \leq n}\left|z_{i}\right|>\sqrt{n} /(\log n)^{2}\right) \\
& -\mathbf{P}\left(|\hat{S} / \sqrt{n}|>t_{p n}, \max _{1 \leq i \leq n}\left|z_{i}\right|>\sqrt{n} /(\log n)^{2}\right)
\end{aligned}
$$

by Lemma 3.3, it remains to prove

$$
\mathbf{P}\left(|\tilde{S} / \sqrt{n}|>t_{p n}, \max _{1 \leq i \leq n}\left|z_{i}\right|>\sqrt{n} /(\log n)^{2}\right)=o\left(p^{-2}\right) \text { with } \tilde{S}=S \text { or } \hat{S}
$$

as $n \rightarrow \infty$. Let us write $\mathbf{Z}_{1}=\left(\left|z_{1}\right|>\sqrt{n} /(\log n)^{2}\right), \mathbf{Z}_{2}=\left(\left|z_{2}\right|>\sqrt{n} /(\log n)^{2}\right)$, $\mathbf{Z}_{2 n}=\left(\max _{2 \leq i \leq n}\left|z_{i}\right|>\sqrt{n} /(\log n)^{2}\right)$. Then, noting $z_{i}=\hat{z}_{i}$ for $2 \leq i \leq n$ if $\mathbf{Z}_{2 n}^{c}$ holds, we have

$$
\begin{aligned}
(3.7) \leq & n \mathbf{P}\left(\mathbf{Z}_{1} \cap \mathbf{Z}_{2 n}\right)+n \mathbf{P}\left(\left(|\tilde{S} / \sqrt{n}|>t_{p n}\right) \cap \mathbf{Z}_{1} \cap \mathbf{Z}_{2 n}^{c}\right) \\
\leq & n^{2} \mathbf{P}\left(\mathbf{Z}_{1} \cap \mathbf{Z}_{2}\right)+n \mathbf{P}\left(\left|z_{1} / \sqrt{n}\right|>\delta t_{p n}\right) \\
& +n \mathbf{P}\left(\left(\left|\sum_{i=2}^{n} z_{i} / \sqrt{n}\right|>(1-\delta) t_{p n}\right) \cap \mathbf{Z}_{1} \cap \mathbf{Z}_{2 n}^{c}\right) \\
\leq & n^{2} \mathbf{P}\left(\mathbf{Z}_{1} \cap \mathbf{Z}_{2}\right)+n \mathbf{P}\left(\left|z_{1} / \sqrt{n}\right|>\delta t_{p n}\right) \\
& +n \mathbf{P}\left(\mathbf{Z}_{1}\right) \mathbf{P}\left(\left|\sum_{i=2}^{n} \hat{z}_{i} / \sqrt{n}\right|>(1-\delta) t_{p n}\right)
\end{aligned}
$$

where $\delta$ can be arbitrarily small. In the above inequality, applying Condition (3.1) and the Chebyshev inequality, we have

$$
n \mathbf{P}\left(\left|z_{1} / \sqrt{n}\right|>\delta t_{p n}\right)=o\left(n^{-2}\right) \text { and } n^{2} \mathbf{P}\left(\mathbf{Z}_{1} \cap \mathbf{Z}_{2}\right)=o\left(n^{-2}\right) .
$$

For the second probability in (3.8), by (3.13) of Lemma 3.3, we have

$$
\mathbf{P}\left(\left|\sum_{i=2}^{n} \hat{z}_{i} / \sqrt{n}\right|>(1-\delta) t_{p n}\right)=o\left(p^{-2+6 \delta}\right)
$$

for $n$ sufficiently large, which combined with

$$
\mathbf{P}\left(\mathbf{Z}_{1}\right) \leq \mathbf{E}\left|z_{1}\right|^{6-\kappa}(\log n)^{12-2 \kappa} / n^{3-\kappa / 2}
$$

shows that, for sufficiently small $\kappa$ and $\delta$,

$$
n \mathbf{P}\left(\mathbf{Z}_{1}\right) \mathbf{P}\left(\left|\sum_{i=2}^{n} \hat{z}_{i} / \sqrt{n}\right|>(1-\delta) t_{p n}\right)=o\left(p^{-2}\right)
$$

as $n \rightarrow \infty$. Hence (3.7) is proved, and we have obtained (3.2).

Now let us prove (3.3). Note that

$$
\sqrt{n}|\mathbf{E} z|=\sqrt{n}|\mathbf{E} \hat{\xi} \hat{\eta}-\mathbf{E} \hat{\xi} \hat{\eta} \mathbf{I}(|\hat{\xi} \hat{\eta}|>\epsilon \sqrt{n})|=o\left(n^{-3 / 2}\right) .
$$

Hence for $n$ sufficiently large,

$$
\mathbf{P}(|S / \sqrt{n}|>A \sqrt{\log p}) \leq \mathbf{P}(|(S-n \mathbf{E} z) / \sqrt{n}|>0.9 A \sqrt{\log p})
$$


So by (3.24) of Lemma 3.5, we have

$$
\begin{aligned}
& \mathbf{P}(|(S-n \mathbf{E} z) / \sqrt{n}|>0.9 A \sqrt{\log p}) \\
& \leq \mathbf{P}\left(\left|T_{z}\right|>0.9 A \sqrt{\log p} / \sqrt{1+\epsilon_{1}}\right)+n^{-M},
\end{aligned}
$$

where $T_{z}=(S-n \mathbf{E} z) / \sqrt{\sum_{i=1}^{n}\left(z_{i}-\mathbf{E} z_{i}\right)^{2}}$ and $M>A^{2} / 4$. Now applying Theorem 2.3 of Jing, Shao and Wang [8],

$$
\mathbf{P}\left(\left|T_{z}\right|>0.9 A \sqrt{\log p} / \sqrt{1+\epsilon_{1}}\right) /\left(2\left(1-\Phi\left(0.9 A \sqrt{\log p} / \sqrt{1+\epsilon_{1}}\right)\right)\right) \rightarrow 1
$$

as $n \rightarrow \infty$, which combined with (3.10), (3.11) and the fact $1-\Phi(s) \sim \frac{1}{\sqrt{2 \pi} s} \mathrm{e}^{-s^{2} / 2}$ as $s \rightarrow \infty$, implies (3.3).

Lemma 3.3. For the $\hat{z}_{i}, i=1,2, \ldots, n$, we have

$$
\mathbf{P}\left(|\hat{S} / \sqrt{n}|>t_{p}\right) /\left(\frac{e^{-y / 2}}{\sqrt{2 \pi}} p^{-2}\right) \rightarrow 1,
$$

and for sufficiently small but fixed $\delta>0$,

$$
\mathbf{P}\left(\left|\sum_{i=2}^{n} \hat{z}_{i} / \sqrt{n}\right|>(1-\delta) t_{p n}\right)=o\left(p^{-2+6 \delta}\right),
$$

as $n \rightarrow \infty$.

Proof. The proofs of (3.12) and (3.13) are the same. We only prove (3.12). Note that

$$
\begin{aligned}
\sqrt{n}|\mathbf{E} \hat{z}| & =\sqrt{n}\left|\mathbf{E} \hat{\xi} \hat{\eta}-\mathbf{E} \hat{\xi} \hat{\eta} \mathbf{I}(|\hat{\xi} \hat{\eta}|>\epsilon \sqrt{n})-\mathbf{E} z \mathbf{I}\left(|z|>\sqrt{n} /(\log n)^{2}\right)\right| \\
& =o\left(n^{-3 / 2}\right)
\end{aligned}
$$

as $n \rightarrow \infty$ by the inequality $\mathbf{E}|z| \mathbf{I}(|z|>x) \leq x^{-5+\kappa} \mathbf{E}|z|^{6-\kappa}$ and Lemma 3.1. Hence in order to show (3.12), it suffices to prove

$$
\mathbf{P}\left(|(\hat{S}-n \mathbf{E} \hat{z}) / \sqrt{n}|>t_{p n}\right) /\left(\frac{e^{-y / 2}}{\sqrt{2 \pi}} p^{-2}\right) \rightarrow 1
$$

as $n \rightarrow \infty$. Applying (3.21) of Lemma 3.5, we have

$$
\begin{aligned}
& \mathbf{P}\left(\left|T_{\hat{z}}\right|>\left(1+(\log n)^{-2}\right)^{1 / 2} t_{p n}\right)-n^{-M} \\
& \quad \leq \mathbf{P}\left(|(\hat{S}-n \mathbf{E} \hat{z}) / \sqrt{n}|>t_{p n}\right) \\
& \quad \leq \mathbf{P}\left(\left|T_{\hat{z}}\right|>\left(1-(\log n)^{-2}\right)^{1 / 2} t_{p n}\right)+n^{-M},
\end{aligned}
$$

where $T_{\hat{z}}=(\hat{S}-n \mathbf{E} \hat{z}) / \sqrt{\sum_{i=1}^{n}\left(\hat{z}_{i}-\mathbf{E} \hat{z}\right)^{2}}$ and $M>3$. Note that

$$
\left(1 \pm(\log n)^{-2}\right) t_{p n}^{2}=t_{p n}^{2}+o(1)
$$

as $n \rightarrow \infty$. So in order to get (3.15), we need to prove

$$
\mathbf{P}\left(\left|T_{\tilde{z}}\right|>t_{p n}\right) /\left(\frac{e^{-y / 2}}{\sqrt{2 \pi}} p^{-2}\right) \rightarrow 1
$$

as $n \rightarrow \infty$. Now we can invoke Theorem 2.3 of Jing, Shao and Wang 8 to obtain (3.16). Hence, we have completed the proof of (3.12). 
The following Lemma 3.4 is useful when we estimate $b_{2}$ of Lemma 2.1

Lemma 3.4. For the above truncated random variables,

$$
\mathbf{P}\left(\left|\left(S+S^{\prime}-n \overline{\hat{\xi}}\left(\overline{\hat{\eta}}+\overline{\hat{\eta}}^{\prime}\right)\right) / \sqrt{n}\right|>2 t_{p}\right)=o\left(p^{-3}\right)
$$

and

$$
\mathbf{P}\left(\left|\left(S-S^{\prime}-n \overline{\hat{\xi}}\left(\overline{\hat{\eta}}-\overline{\hat{\eta}}^{\prime}\right)\right) / \sqrt{n}\right|>2 t_{p}\right)=o\left(p^{-3}\right)
$$

as $n \rightarrow \infty$

Proof. Similarly as in the derivation of (3.4), and noting (3.9), in order to prove (3.17), we need to prove

$$
\mathbf{P}\left(\left|\left(S+S^{\prime}-n\left(\mathbf{E} z+\mathbf{E} z^{\prime}\right)\right) / \sqrt{n}\right|>2 t_{p n}\right)=o\left(p^{-3}\right)
$$

as $n \rightarrow \infty$. By (3.22) of Lemma 3.5. we have

$\mathbf{P}\left(\left|\left(S+S^{\prime}-n\left(\mathbf{E} z+\mathbf{E} z^{\prime}\right)\right)\right|>2 t_{p n}\right) \leq \mathbf{P}\left(\left|T_{z z^{\prime}}\right|>\left(1-\epsilon_{1} / 2\right)^{1 / 2} \sqrt{2} t_{p n}\right)+n^{-M}$,

where $T_{z z^{\prime}}=\left(S+S^{\prime}-n\left(\mathbf{E} z+\mathbf{E} z^{\prime}\right)\right) /\left(\sum_{i=1}^{n}\left(z_{i}+z_{i}^{\prime}-\mathbf{E} z_{i}-\mathbf{E} z_{i}^{\prime}\right)^{2}\right)^{1 / 2}$ and $M>4$. Now applying Theorem 2.3 of Jing, Shao and Wang [] to

$$
\mathbf{P}\left(\left|T_{z z^{\prime}}\right|>\left(1-\epsilon_{1} / 2\right)^{1 / 2} \sqrt{2} t_{p n}\right)
$$

gives (3.19). So the proof of (3.17) is complete, and we can get (3.18) similarly.

Lemma 3.5 guarantees that in the proof of Theorem 1.1, the sample variance and the population variance are equivalent.

Lemma 3.5. For the above truncated random variables $\hat{\xi}, z, z^{\prime}, \hat{\xi}_{i}, z_{i}, z_{i}^{\prime}, i=$ $1,2, \ldots, n$, we have

$$
\begin{aligned}
& \mathbf{P}\left(\left|\sum_{i=1}^{n} \hat{\xi}_{i}^{2} / n-\overline{\hat{\xi}}^{2}-1\right|>1 /(\log n)^{2}\right) \leq n^{-M}, \\
& \mathbf{P}\left(\left|\sum_{i=1}^{n}\left(\hat{z}_{i}-\mathbf{E} \hat{z}\right)^{2} / n-1\right|>1 /(\log n)^{2}\right) \leq n^{-M}, \\
& \mathbf{P}\left(\left|\frac{1}{n} \sum_{i=1}^{n}\left(z_{i}+z_{i}^{\prime}-\mathbf{E} z_{i}-\mathbf{E} z_{i}^{\prime}\right)^{2}-2\right|>\epsilon_{1}\right) \leq n^{-M}, \\
& \mathbf{P}\left(\left|\frac{1}{n} \sum_{i=1}^{n}\left(z_{i}-z_{i}^{\prime}-\mathbf{E} z_{i}+\mathbf{E} z_{i}^{\prime}\right)^{2}-2\right|>\epsilon_{1}\right) \leq n^{-M}, \\
& \mathbf{P}\left(\left|\frac{1}{n} \sum_{i=1}^{n}\left(z_{i}-\mathbf{E} z_{i}\right)^{2}-1\right|>\epsilon_{1}\right) \leq n^{-M},
\end{aligned}
$$

with arbitrarily large $M$ (say, greater than 100) and arbitrarily small but fixed $\epsilon_{1}$.

Proof. The Bernstein inequality implies that

$$
\mathbf{P}\left(|\overline{\hat{\xi}}-\mathbf{E} \hat{\xi}|>n^{-1 / 6}\right) \leq n^{-M} .
$$


Noting

$$
\begin{aligned}
1 & \geq \mathbf{E} \hat{\xi}^{2} \\
& =1-\mathbf{E} \xi^{2} \mathbf{I}\left(|\xi|>d_{n}\right) \geq 1-n^{-(4-\kappa) / 3} \mathbf{E}|\xi|^{6-\kappa}
\end{aligned}
$$

and $|\mathbf{E} \hat{\xi}| \leq n^{-(5-\kappa) / 3} \mathbf{E}|\xi|^{6-\kappa}$, we have

$$
\begin{aligned}
& \mathbf{P}\left(\left|\sum_{i=1}^{n} \hat{\xi}_{i}^{2} / n-\overline{\hat{\xi}}^{2}-1\right|>1 /(\log n)^{2}\right) \\
& \leq \mathbf{P}\left(\left|\sum_{i=1}^{n} \hat{\xi}_{i}^{2} / n-\mathbf{E} \hat{\xi}^{2}\right|>1 /\left(2(\log n)^{2}\right)\right)+n^{-M},
\end{aligned}
$$

for $n$ sufficiently large. For the probability term in (3.25), we have

$$
\begin{aligned}
& \mathbf{P}\left(\left|\sum_{i=1}^{n} \hat{\xi}_{i}^{2} / n-\mathbf{E} \hat{\xi}^{2}\right|>1 /\left(2(\log n)^{2}\right)\right) \\
\leq & \exp \left(-\frac{t}{2(\log n)^{2}}\right)\left(\mathbf{E} \exp \left(t n^{-1}\left(\hat{\xi}^{2}-\mathbf{E} \hat{\xi}^{2}\right)\right)\right)^{n} \\
& \quad+\exp \left(-\frac{t}{2(\log n)^{2}}\right)\left(\mathbf{E} \exp \left(t n^{-1}\left(\mathbf{E} \hat{\xi}^{2}-\hat{\xi}^{2}\right)\right)\right)^{n} \\
\leq & 2 \exp \left(-\frac{t}{2(\log n)^{2}}\right)\left(1+\frac{1}{2} t^{2} n^{-2} \mathbf{E} \xi^{4} \exp \left(2 t /(\log n)^{4}\right)\right)^{n} \\
\leq & n^{-M}, \quad\left(\operatorname{let} t=2 M \log ^{3} n\right)
\end{aligned}
$$

where the above second inequality follows from the Taylor expansion of the exponential function and the fact that $\hat{\xi}$ is bounded by $d_{n}\left(\leq \sqrt{n} /(\log n)^{2}\right)$, and the last one follows from the fact that $1+x \leq e^{x}$ for $x>0$. Hence we can complete the proof of (3.20).

Now let us prove (3.21). Recalling that $z=\hat{\xi} \hat{\eta} \mathbf{I}(|\hat{\xi} \hat{\eta}| \leq \epsilon \sqrt{n})$, we have

$$
z+\xi \mathbf{I}\left(|\xi|>d_{n}\right) \hat{\eta} \mathbf{I}(|\hat{\xi} \hat{\eta}| \leq \epsilon \sqrt{n})+\xi \eta \mathbf{I}\left(|\eta|>d_{n}\right) \mathbf{I}(|\hat{\xi} \hat{\eta}| \leq \epsilon \sqrt{n})
$$

$$
=\xi \eta-\xi \eta \mathbf{I}(|\xi \eta|>\epsilon \sqrt{n})-\xi \eta \mathbf{I}_{2}-\xi \eta \mathbf{I}_{3}-\xi \eta \mathbf{I}_{4}
$$

where

$$
\begin{aligned}
& \mathbf{I}_{2}=\mathbf{I}\left(|\xi \eta| \mathbf{I}\left(|\xi|>d_{n},|\eta| \leq d_{n}\right) \leq \epsilon \sqrt{n}\right) \\
& \mathbf{I}_{3}=\mathbf{I}\left(|\xi \eta| \mathbf{I}\left(|\xi| \leq d_{n},|\eta|>d_{n}\right) \leq \epsilon \sqrt{n}\right)
\end{aligned}
$$

and

$$
\mathbf{I}_{4}=\mathbf{I}\left(|\xi \eta| \mathbf{I}\left(|\xi|>d_{n},|\eta|>d_{n}\right) \leq \epsilon \sqrt{n}\right)
$$

For the last two terms in (3.26), noting $\mathbf{E} \xi^{2}=\mathbf{E} \eta^{2}=1$, we have

$$
\begin{aligned}
& E\left(\xi \mathbf{I}\left(|\xi|>d_{n}\right) \hat{\eta} \mathbf{I}(|\hat{\xi} \hat{\eta}| \leq \epsilon \sqrt{n})\right)^{2} \\
& \leq \mathbf{E} \xi^{2} \mathbf{I}\left(|\xi|>d_{n}\right) \leq n^{-(4-\kappa) / 3} \mathbf{E}|\xi|^{6-\kappa},
\end{aligned}
$$


and

$$
\mathbf{E}\left(\xi \eta \mathbf{I}\left(|\eta|>d_{n}\right) \mathbf{I}(|\hat{\xi} \hat{\eta}| \leq \epsilon \sqrt{n})\right)^{2} \leq n^{-(4-\kappa) / 3} \mathbf{E}|\eta|^{6-\kappa}
$$

For the last three terms in (3.27), we have

$$
\begin{aligned}
\mathbf{E} \xi^{2} \eta^{2} \mathbf{I}_{2} & \leq \mathbf{E} \xi^{2} \mathbf{I}\left(|\xi|>d_{n}\right) \eta^{2} \mathbf{I}\left(|\eta| \leq d_{n}\right) \\
& \leq n^{-(4-\kappa) / 3} \mathbf{E}|\xi|^{6-\kappa},
\end{aligned}
$$

and

$$
\mathbf{E} \xi^{2} \eta^{2} \mathbf{I}_{3} \leq n^{-(4-\kappa) / 3} \mathbf{E}|\eta|^{6-\kappa}, \quad \mathbf{E} \xi^{2} \eta^{2} \mathbf{I}_{4}=0
$$

for $n$ sufficiently large. Combining (3.26) - (3.31) and making use of the elementary fact

$$
\mathbf{E} a^{2}+\mathbf{E} b^{2}-2 \sqrt{\mathbf{E} a^{2} \mathbf{E} b^{2}} \leq \mathbf{E}(a+b)^{2} \leq \mathbf{E} a^{2}+\mathbf{E} b^{2}+2 \sqrt{\mathbf{E} a^{2} \mathbf{E} b^{2}}
$$

we have

$$
\mathbf{E} z^{2}=1+O(1 / \sqrt{n})
$$

But

$$
\begin{aligned}
\mathbf{E}(\hat{z}-\mathbf{E} \hat{z})^{2} & =\mathbf{E} z^{2}-\mathbf{E} z^{2} \mathbf{I}\left(|z|>\sqrt{n} /(\log n)^{2}\right)-(\mathbf{E} \hat{z})^{2} \\
& =1+O(1 / \sqrt{n})
\end{aligned}
$$

as $n \rightarrow \infty$, where the last equality comes from (3.14) and (3.32). Hence we can repeat the proof of (3.20) to get (3.21).

Finally we will prove (3.22). The proofs of (3.23) and (3.24) are the same as that of (3.22). Noting (3.32) and the basic inequality

$$
(\mathbf{E} a)^{2} \leq \mathbf{E} a^{2}
$$

we have

$$
\mathbf{E}\left(z+z^{\prime}-\mathbf{E} z-\mathbf{E} z^{\prime}\right)^{2}=2-2 \mathbf{E} z z^{\prime}+O(1 / \sqrt{n}) .
$$

Let us turn to the calculation of $\mathbf{E} z z^{\prime}$. Recalling the definitions of $z$ and $z^{\prime}$, we have

$$
\begin{aligned}
z z^{\prime} & +\hat{\xi}^{2} \hat{\eta} \hat{\eta}^{\prime} \mathbf{I}(|\hat{\xi} \hat{\eta}|>\epsilon \sqrt{n}) \mathbf{I}\left(\left|\hat{\xi} \hat{\eta}^{\prime}\right| \leq \epsilon \sqrt{n}\right) \\
& +\hat{\xi}^{2} \hat{\eta} \hat{\eta}^{\prime} \mathbf{I}(|\hat{\xi} \hat{\eta}| \leq \epsilon \sqrt{n}) \mathbf{I}\left(\left|\hat{\xi} \hat{\eta}^{\prime}\right|>\epsilon \sqrt{n}\right) \\
& +\hat{\xi}^{2} \hat{\eta} \hat{\eta}^{\prime} \mathbf{I}(|\hat{\xi} \hat{\eta}|>\epsilon \sqrt{n}) \mathbf{I}\left(\left|\hat{\xi} \hat{\eta}^{\prime}\right|>\epsilon \sqrt{n}\right)=\hat{\xi}^{2} \hat{\eta} \hat{\eta}^{\prime}
\end{aligned}
$$

which implies

$$
\mathbf{E} z z^{\prime}=O\left(n^{-1 / 2}\right)
$$

as $n \rightarrow \infty$. Hence

$$
\mathbf{E}\left(z+z^{\prime}-\mathbf{E} z-\mathbf{E} z^{\prime}\right)^{2}=2+O(1 / \sqrt{n})
$$


Write $\omega_{i}=\left(z_{i}+z_{i}^{\prime}-\mathbf{E} z_{i}-\mathbf{E} z_{i}^{\prime}\right)^{2}, i=1, \ldots, n$. We have $\left|\omega_{i}\right| \leq 16 \epsilon^{2} n$ and $\mathbf{E} \omega_{i}=$ $2+O(1 / \sqrt{n})$. Hence for $n$ large enough,

$$
\begin{aligned}
\mathbf{P}\left(\left|\sum_{i=1}^{n} \omega_{i} / n-2\right|>\epsilon_{1}\right) \leq & \mathbf{P}\left(\left|\sum_{i=1}^{n}\left(\omega_{i}-\mathbf{E} \omega_{i}\right) / n\right|>\epsilon_{1} / 2\right) \\
\leq & \exp \left(-t \epsilon_{1} / 2\right)\left(\mathbf{E} \exp \left(t\left(\omega_{1}-\mathbf{E} \omega_{1}\right) / n\right)\right)^{n} \\
& \quad+\exp \left(-t \epsilon_{1} / 2\right)\left(\mathbf{E} \exp \left(t\left(\mathbf{E} \omega_{1}-\omega_{1}\right) / n\right)\right)^{n} \\
\leq & 2 \exp \left(-t \epsilon_{1} / 2\right) \exp \left(\frac{1}{2} t^{2} n^{-1} \mathbf{E} \omega_{1}^{2} \exp \left(32 \epsilon^{2} t\right)\right) \\
\leq & n^{-M}, \quad\left(\text { let } t=3 M \log n / \epsilon_{1}\right)
\end{aligned}
$$

where $\epsilon$ satisfies

$$
96 M \epsilon^{2} / \epsilon_{1}<1
$$

The following lemma is a special case of Theorem 2.1 in Seoh, Ralescu and Puri 12 .

Lemma 3.6. For Spearman's correlation coefficient (1.8), $1 \leq i<j \leq p$, if all the entries of $\mathcal{X}_{n}$ are continuous random variables, we have as $n \rightarrow \infty$, uniformly in the range $0<x \leq c_{n} n^{1 / 6}, c_{n}=o(1)$,

$$
\mathbf{P}\left(\frac{\left|r_{i j}\right|}{\sqrt{\operatorname{Var} r_{i j}}}>x\right)=2(1-\Phi(x))(1+o(1)),
$$

where $\operatorname{Var} r_{i j}=1 /(n-1)$.

\section{EXAmple}

Now let us give an example explaining that if

$$
\lim _{x \rightarrow \infty} x^{6} \mathbf{P}\left(\left|X X^{\prime}\right|>x\right)>0
$$

then (1.5) may not hold, or the limit could be different. So our weak moment condition (1.6) seems to be optimal for (1.5). In this section, the results are not rigorous.

Example 1. Let $X$ be a symmetric random variable such that

$$
\mathbf{P}(X=0)=1-2 c_{0} a^{-6} \log ^{-1 / 2} a, \mathbf{P}(X>x)=c_{0} x^{-6} \log ^{-1 / 2} x, x>a>1,
$$

where $c_{0}$ is determined by $\mathbf{E} X^{2}=1$. The density function of the absolute continuous part of $X$ is

$$
f(x)=6 c_{0}|x|^{-7} \log ^{-1 / 2}|x|+2^{-1} c_{0}|x|^{-7} \log ^{-3 / 2}|x|, \quad|x|>a .
$$


Let $X^{\prime}, X_{k i}, 1 \leq k \leq n, 1 \leq i \leq p$, be i.i.d. random variables with the same distribution as $X$. Then for $x>a^{2}$,

$$
\begin{aligned}
\mathbf{P}(\mid & \left.X X^{\prime} \mid>x\right)=4 \int_{a}^{x / a} \mathbf{P}\left(X>x / x_{1}\right) f\left(x_{1}\right) d x_{1}+4 \mathbf{P}(X>a) \mathbf{P}\left(X^{\prime}>x / a\right) \\
= & 24 c_{0}^{2} x^{-6} \int_{(\log a) / \log x}^{1-(\log a) / \log x} y^{-1 / 2}(1-y)^{-1 / 2} d y \\
& +2 c_{0}^{2} x^{-6} \log ^{-1} x \int_{(\log a) / \log x}^{1-(\log a) / \log x} y^{-3 / 2}(1-y)^{-1 / 2} d y \\
& +4 \mathbf{P}(X>a) \mathbf{P}\left(X^{\prime}>x / a\right),
\end{aligned}
$$

which implies that

$$
\lim _{x \rightarrow \infty} x^{6} \mathbf{P}\left(\left|X X^{\prime}\right|>x\right)=24 c_{0}^{2} \int_{0}^{1} y^{-1 / 2}(1-y)^{-1 / 2} d y .
$$

Note that (4.3) is weaker than the moment condition (1.6).

Choose $p=n$. We will use an indirect argument, not so rigorously, to show that for random variables $X_{k i}, 1 \leq k \leq n, 1 \leq i \leq p$,

$$
\mathbf{P}\left(n L_{n}^{2} \leq t_{p}^{2}\right) \nrightarrow \exp \left(-K e^{-y / 2}\right)
$$

as $n \rightarrow \infty$.

Now we assume for random variables $X_{k i}, 1 \leq k \leq n, 1 \leq i \leq p$, that

$$
\mathbf{P}\left(n L_{n}^{2} \leq t_{p}^{2}\right) \rightarrow \exp \left(-K e^{-y / 2}\right)
$$

as $n \rightarrow \infty$. Based on the assumption (4.4), we may get a contradiction.

Define

$$
\tilde{X}_{k i}=X_{k i} \mathbf{I}\left(\left|X_{k i}\right| \leq n^{1 / 3}\right), \tilde{\mathbf{X}}_{i}=\left(\tilde{X}_{1 i}, \ldots, \tilde{X}_{n i}\right)^{T}, \overline{\tilde{X}_{i}}=\sum_{k=1}^{n} \tilde{X}_{k i} / n,
$$

and

$$
\tilde{\rho}_{i j}=\frac{\left(\tilde{\mathbf{X}}_{i}-\overline{\tilde{X}_{i}}\right)^{T}\left(\tilde{\mathbf{X}}_{j}-\overline{\tilde{X}_{j}}\right)}{\left\|\tilde{\mathbf{X}}_{i}-\overline{\tilde{X}_{i}}\right\| \|\left(\tilde{\mathbf{X}}_{j}-\overline{\tilde{X}_{j}} \|\right.}, \tilde{L}_{n}=\max _{1 \leq i<j \leq p}\left|\tilde{\rho}_{i j}\right|,
$$

where $1 \leq i<j \leq p, 1 \leq k \leq n$. Then,

$$
\begin{aligned}
\left|\mathbf{P}\left(n L_{n}^{2} \leq t_{p}^{2}\right)-\mathbf{P}\left(n \tilde{L}_{n}^{2} \leq t_{p}^{2}\right)\right| & \leq \mathbf{P}\left(\max _{1 \leq k \leq n, 1 \leq i \leq p}\left|X_{k i}\right|>n^{1 / 3}\right) \\
& \leq n p \mathbf{P}\left(\left|X_{12}\right|>n^{1 / 3}\right) \\
& \rightarrow 0
\end{aligned}
$$

as $n \rightarrow \infty$. Write

$$
\tilde{L}_{1 n}=\max _{1 \leq i<j \leq p}\left|\frac{\left(\tilde{\mathbf{X}}_{i}-\overline{\tilde{X}_{i}}\right)^{T}\left(\tilde{\mathbf{X}}_{j}-\overline{\tilde{X}_{j}}\right)}{n\left(1-1 /(\log n)^{2}\right)}\right|
$$

and

$$
\tilde{L}_{2 n}=\max _{1 \leq i<j \leq p}\left|\frac{\left(\tilde{\mathbf{X}}_{i}-\overline{\tilde{X}_{i}}\right)^{T}\left(\tilde{\mathbf{X}}_{j}-\overline{\tilde{X}_{j}}\right)}{n\left(1+1 /(\log n)^{2}\right)}\right| .
$$


By similar arguments leading to (2.2), we have

$$
\begin{aligned}
& \mathbf{P}\left(n \tilde{L}_{1 n}^{2} \leq t_{p}^{2}\right)-\mathbf{P}\left(\max _{1 \leq i \leq p}\left|\left\|\tilde{\mathbf{X}}_{i}-\overline{\tilde{X}_{i}}\right\|^{2}-n\right|>n /(\log n)^{2}\right) \\
\leq & \mathbf{P}\left(n \tilde{L}_{n}^{2} \leq t_{p}^{2}\right) \\
\leq & \mathbf{P}\left(n \tilde{L}_{2 n}^{2} \leq t_{p}^{2}\right)+\mathbf{P}\left(\max _{1 \leq i \leq p}\left|\left\|\tilde{\mathbf{X}}_{i}-\overline{\tilde{X}_{i}}\right\|^{2}-n\right|>n /(\log n)^{2}\right) .
\end{aligned}
$$

By (3.20) of Lemma 3.5,

$$
\mathbf{P}\left(\max _{1 \leq i \leq p}\left|\left\|\tilde{\mathbf{X}}_{i}-\overline{\tilde{X}_{i}}\right\|^{2}-n\right|>n /(\log n)^{2}\right) \leq p n^{-M} \rightarrow 0
$$

as $n \rightarrow \infty$. Also note that

$$
t_{p}^{2}\left(1 \pm 1 /(\log n)^{2}\right)=t_{p}^{2}+o(1)
$$

as $n \rightarrow \infty$. Combining (4.5)-(4.7) and the assumption (4.4) gives

$$
\mathbf{P}\left(n \overline{\tilde{L}}_{n}^{2} \leq t_{p}^{2}\right) \rightarrow \exp \left(-K e^{-y / 2}\right)
$$

as $n \rightarrow \infty$, where

$$
\overline{\tilde{L}}_{n}=\max _{1 \leq i<j \leq p}\left|\left(\tilde{\mathbf{X}}_{i}-\overline{\tilde{X}}_{i}\right)^{T}\left(\tilde{\mathbf{X}}_{j}-\overline{\tilde{X}_{j}}\right) / n\right| .
$$

Now for $\epsilon$ satisfying (3.33), define

$$
\begin{gathered}
\tilde{\xi}_{k i j}=\tilde{X}_{k i} \tilde{X}_{k j} \mathbf{I}\left(\left|\tilde{X}_{k i} \tilde{X}_{k j}\right| \leq \epsilon \sqrt{n}\right), 1 \leq k \leq n, \\
\tilde{y}_{i j}=\frac{\sum_{k=1}^{n} \xi_{k i j}-n \overline{\tilde{X}_{i}} \overline{\tilde{X}_{j}}}{\sqrt{n}}, 1 \leq i<j \leq p,
\end{gathered}
$$

and

$$
\tilde{W}_{n}=\max _{1 \leq i<j \leq p}\left|\tilde{y}_{i j}\right| .
$$

Now following the same proof of (2.8), we have

$$
\mathbf{P}\left(n \tilde{W}_{n}^{2} \leq t_{p}^{2}\right) \rightarrow \exp \left(-K e^{-y / 2}\right)
$$

as $n \rightarrow \infty$. Noting (4.8), we have

$$
\mathbf{P}\left(n \overline{\tilde{L}}_{n}^{2} \leq t_{p}^{2}\right)-\mathbf{P}\left(n \tilde{W}_{n}^{2} \leq t_{p}^{2}\right) \rightarrow 0
$$

as $n \rightarrow \infty$. Using the inequality $\mathbf{P}\left(\bigcup_{i} \Theta_{i}\right) \geq \sum_{i} \mathbf{P}\left(\Theta_{i}\right)-\sum_{i \neq j} \mathbf{P}\left(\Theta_{i} \cap \Theta_{j}\right)$, we conjecture

$$
\begin{aligned}
\mid \mathbf{P}\left(n \overline{\tilde{L}}_{n}^{2} \leq t_{p}^{2}\right) & -\mathbf{P}\left(n \tilde{W}_{n}^{2} \leq t_{p}^{2}\right) \mid \\
\geq 2^{-1} n p(p-1) \mathbf{P}\left(\mathbf{L}_{112}\right) & \\
& -n p^{3} \mathbf{P}\left(\mathbf{L}_{112} \cap \mathbf{L}_{113}\right)-(n p(p-1))^{2} \mathbf{P}\left(\mathbf{L}_{112} \cap \mathbf{L}_{212}\right) \\
& -\mathbf{P}\left(n \tilde{\tilde{L}}_{n}^{2}>t_{p}^{2}\right)
\end{aligned}
$$


where $\mathbf{L}_{k i j}=\left(\left|\tilde{X}_{k i} \tilde{X}_{k j}\right|>A_{0} \sqrt{n}\right)$, and $A_{0}$ is a constant specified in (4.20). For (4.13),

$$
\begin{aligned}
n p(p-1) \mathbf{P}\left(\mathbf{L}_{112}\right) & =n p(p-1) \mathbf{P}\left(\left|X_{11} X_{12}\right|>A_{0} \sqrt{n},\left|X_{11}\right| \leq n^{1 / 3},\left|X_{12}\right| \leq n^{1 / 3}\right) \\
& =4 n p(p-1) \int_{A_{0} n^{1 / 6}}^{n^{1 / 3}} \mathbf{P}\left(A_{0} \sqrt{n} / x<X_{12} \leq n^{1 / 3}\right) f(x) d x \\
(4.16) & \rightarrow 24 c_{0}^{2} A_{0}^{-6} c
\end{aligned}
$$

with $c=\int_{1 / 3}^{2 / 3} y^{-1 / 2}(1-y)^{-1 / 2} d y$, as $n \rightarrow \infty$. For the first term in (4.14),

$$
\begin{aligned}
& n p^{3} \mathbf{P}\left(\mathbf{L}_{112} \cap \mathbf{L}_{113}\right) \\
& =n p^{3} \mathbf{P}\left(\left|X_{11} X_{12}\right|>A_{0} \sqrt{n},\left|X_{11} X_{13}\right|>A_{0} \sqrt{n},\left|X_{11}\right| \leq n^{1 / 3},\right. \\
& \left.\quad\left|X_{12}\right| \leq n^{1 / 3},\left|X_{13}\right| \leq n^{1 / 3}\right) \\
& =8 n p^{3} \int_{A_{0} n^{1 / 6}}^{n^{1 / 3}} \mathbf{P}^{2}\left(A_{0} \sqrt{n} / x<X_{12} \leq n^{1 / 3}\right) f(x) d x .
\end{aligned}
$$

Since

$$
\mathbf{P}^{2}\left(A_{0} \sqrt{n} / x<X_{12} \leq n^{1 / 3}\right) \leq 2 c_{0}^{2}\left(\left(A_{0} \sqrt{n} / x\right)^{-12} \log ^{-1}\left(A_{0} \sqrt{n} / x\right)+n^{-4} \log ^{-1} n^{1 / 3}\right),
$$

we have

$$
\begin{aligned}
& \quad 8 n p^{3} \int_{A_{0} n^{1 / 6}}^{n^{1 / 3}} \mathbf{P}^{2}\left(A_{0} \sqrt{n} / x<X_{12} \leq n^{1 / 3}\right) f(x) d x \\
& \leq \quad 16 n p^{3} c_{0}^{2} \int_{A_{0} n^{1 / 6}}^{n^{1 / 3}}\left(A_{0} \sqrt{n} / x\right)^{-12} \log ^{-1}\left(A_{0} \sqrt{n} / x\right) f(x) d x \\
& \quad+16 n p^{3} c_{0}^{2} \int_{A_{0} n^{1 / 6}}^{n^{1 / 3}} n^{-4} \log ^{-1}\left(n^{1 / 3}\right) f(x) d x \\
& \quad I_{1}+I_{2} .
\end{aligned}
$$

For $I_{1}$,

$$
\begin{aligned}
I_{1}= & 16 n p^{3} c_{0}^{3}\left(A_{0} \sqrt{n}\right)^{-12} \int_{A_{0} n^{1 / 6}}^{n^{1 / 3}} \log ^{-1}\left(A_{0} \sqrt{n} / x\right) x^{5}\left(6 \log ^{-1 / 2} x+2^{-1} \log ^{-3 / 2} x\right) d x \\
= & 16 n p^{3} c_{0}^{3}\left(A_{0} \sqrt{n}\right)^{-12} \int_{\log \left(A_{0} n^{1 / 6}\right) / \log \left(A_{0} \sqrt{n}\right)}^{\log \left(n^{1 / 3}\right) / \log \left(A_{0} \sqrt{n}\right)}(1-y)^{-1} \exp \left(6 y \log \left(A_{0} \sqrt{n}\right)\right) \\
& \quad \times\left(6 y^{-1 / 2} \log ^{-1 / 2}\left(A_{0} \sqrt{n}\right)+2^{-1} y^{-3 / 2} \log ^{-3 / 2}\left(A_{0} \sqrt{n}\right)\right) d x \\
= & o \text { (1) }
\end{aligned}
$$

as $n \rightarrow \infty$. For $I_{2}$, we also have $I_{2}=o(1)$ as $n \rightarrow \infty$. Hence

$$
n p^{3} \mathbf{P}\left(\mathbf{L}_{112} \cap \mathbf{L}_{113}\right)=o(1)
$$

as $n \rightarrow \infty$.

For the second term in (4.14),

$$
(n p(p-1))^{2} \mathbf{P}\left(\mathbf{L}_{112} \cap \mathbf{L}_{212}\right) \rightarrow\left(24 c_{0}^{2} A_{0}^{-6} c\right)^{2}
$$

as $n \rightarrow \infty$. 
Hence combining (4.12)-(4.18) and our assumption (4.4), we have

$$
\begin{aligned}
0 & =\lim _{n \rightarrow \infty}\left(\mathbf{P}\left(n \overline{\tilde{L}}_{n}^{2} \leq t_{p}^{2}\right)-\mathbf{P}\left(n \tilde{W}_{n}^{2} \leq t_{p}^{2}\right)\right) \\
& \geq 12 c_{0}^{2} A_{0}^{-6} c-\left(24 c_{0}^{2} A_{0}^{-6} c\right)^{2}-\left(1-\exp \left(-K e^{-y / 2}\right)\right) .
\end{aligned}
$$

Let

$$
A_{0}=\max \left(\left(48 c_{0}^{2} c\right)^{1 / 6}, \epsilon\right)
$$

Select $y$ so that

$$
12 c_{0}^{2} A_{0}^{-6} c-\left(24 c_{0}^{2} A_{0}^{-6} c\right)^{2}>1-\exp \left(-K e^{-y / 2}\right) .
$$

Then (4.19) yields $0>0$, a contradiction if we have (4.13)-(4.15). Hence for the random variable (4.2), we may not have (4.4).

\section{ACKNOWLEDGMENTS}

The author would like to thank the referee for his/her valuable suggestions and remarks.

\section{REFERENCES}

[1] Arratia, R., Goldstein, L. and Gordon, L. (1989). Two moments suffice for Poisson approximation: the Chen-Stein method. Ann. Prob., 17, 9-25. MR972770 (90b:60021)

[2] Barbour, A. and Eagleson, G. (1984). Poisson convergence for dissociated statistics. J. R. Statist. Soc. B, 46, 397-402. MR790624 (86k:60033)

[3] Fisher, R. A. (1915). Frequency distribution of the values of the correlation coefficient in samples from an indefinitely large population. Biometrika, 10, 507-521.

[4] Hájek, J., Šidák, Z. and Sen, P. K. (1999). Theory of Rank Tests. Academic Press, New York.

[5] Hollander, M. and Wolfe, D. A. (1999). Nonparametric Statistical Methods. Wiley, New York. MR.1666064 (99m:62004)

[6] Hotelling, H. (1953). New light on the correlation coefficient and its transforms. (with discussion) J. Roy. Statist. Soc. Ser. B., 15, 193-232. MR0060794 (15:728d)

[7] Jiang, T. (2004). The asymptotic distributions of the largest entries of sample correlation matrices. Ann. Appl. Prob., 14, 865-880. MR2052906 (2005b:60053)

[8] Jing, B.-Y., Shao, Q.-M. and Wang, Q. (2003). Self-normalized Cramér-type large deviations for independent random variables. Ann. Probab., 31, 2167-2215. MR2016616 (2004k:60069)

[9] Johnstone, I. (2001). On the distribution of the largest eigenvalue in principal component analysis. Ann. Stat., 29, 295-327. MR.1863961(2002i:62115)

[10] Shao, Q.-M. (1997). Self-normalized large deviations. Ann. Probab., 25, 285-328. MR 1428510 (98b:60056)

[11] Shao, Q.-M. (1999). A Cramér type large deviation result for Student's t-statistic. J. Theoret. Probab., 12, 385-398. MR1684750 (2000d:60046)

[12] Seoh, M., Ralescu, S. and Puri, M. L. (1985). Cramér type large deviations for generalized rank statistics. Ann. Prob., 13, 115-125. MR770632 (86k:62077)

[13] Spearman, C. (1904). The proof and measurement of association between two things. Amer. J. Psychol., 15, 72-101.

[14] Wang, Q. Y. and Jing, B.-Y. (1999). An exponential non-uniform Berry-Esséen bound for self-normalized sums. Ann. Probab., 27, 2068-2088. MR1742902 (2001c:60045)

Department of Statistics and Applied Probability, National University of SingaPORE, SingaPORE, 117546

E-mail address: stazw@nus.edu.sg 